\title{
MEASUREMENT OF ELECTROMAGNETIC CROSS SECTIONS IN HEAVY ION INTERACTIONS AND ITS CONSEQUENCES FOR LUMINOSITY LIFETIMES IN ION COLLIDERS
}

\author{
P. Grafström ${ }^{*}$, CERN, CH, S. Datz, H.F. Krause, C.R. Vane, Oak Ridge, TN, U.S., H. Knudsen, \\ U. Mikkelsen, Aarhus Univ., Aarhus, DK, R.H. Schuch, MSL, Stockholm, S, C. Scheidenberger, GSI, \\ Darmstadt, D, Z. Vilakazi, Witwatersland Univ. Johannesburg, S. Africa.
}

\begin{abstract}
The limitation of the luminosity lifetime in high energy heavy ion colliders like RHIC or LHC operating in ion mode is set by the very large cross section of beam - beam interactions. One of the dominant processes at relativistic energies is electron capture from pair production in the strong electromagnetic field provided by the high $Z$ of the ions. The capture cross sections for $\mathrm{Pb}^{\text {s2t }}$ interacting with a number targets have been measured using one of the high energy resolution $158 \mathrm{GeV} /$ nucleon beams at CERN. The results, together with results on electromagnetic dissociation, are discussed in terms of beam lifetimes for RHIC and LHC using extrapolations of the measurements to the corresponding collider energies
\end{abstract}

\section{INTRODUCTION}

One of the principal limitations to the luminosity lifetime in high energy heavy ion colliders is the loss of beam particles due to the large cross section for beam-beam interactions. At very high energies there are essentially three processes that contribute to the losses: Electron capture, electromagnetic dissociation and hadronic processes.

The electron capture proceeds through several different mechanisms. In Radiative Electron Capture (REC) the ion picks up a target electron and simultaneously emits a photon. The capture can also be non-radiative (NRC) when an electron bound to an atom is picked up. However at very high energies a third mechanism is dominant. Electronpositron pairs are created in the strong electromagnetic pulse produced when the ion passes near the target nucleus and with a certain probability the electron can be captured (ECPP; Electron Capture via Pair Production). This later process, contrary to the two others, does not require an electron in the initial state, and thus the reaction can take place between two initially bare ions. This fact, together with the large cross section, which increases with energy, makes ECPP a potential limiting factor for high energy heavy ion colliders.

The strong electromagnetic field between the ions is also the origin of a possible nuclear dissociation of one or both of the ions in the interaction. The electromagnetic dissociation proceeds via photon excitation of the nucleus followed by decay through particle emission. These electromagnetic processes dominate at large impact parameters of the collisions. However contributions from hadronic interactions in more central collisions are not negligible even for very relativistic energies.

In this paper we report measurements on the total cross section for electron capture and measurements of the sum the total nuclear loss cross section through electromagnetic dissociation and hadronic interactions.

The experiment was performed at the CERN Super Proton Synchrotron (SPS) with completely stripped $\mathrm{Pb}^{82+}$ ions at $33 \mathrm{TeV}(158 \mathrm{GeV} / \mathrm{A})$. These measurements were mainly motivated by their theoretical importance as a test ground of QED under nonperturbative conditions. However, using the data and extrapolating to the energies of the RHIC and LHC colliders, limits on the beam lifetimes can also be estimated. Similar estimates have been done previously $[1,2,3,4]$, though our limits are based upon the first measurements made in an energy regime where the simple reliable scaling laws should be valid [5].

\section{DESCRIPTION OF THE EXPERIMENT}

The SPS external beam lines are used to transport the $33 \mathrm{TeV} \mathrm{Pb}{ }^{82+}$ beam from the extraction points, via magnetic beam splitters, to a number of different experiments located between $500 \mathrm{~m}$ and $1 \mathrm{~km}$ away. The beam lines are comprised of a large number of bending magnets and quadrupoles. In our application we used the whole system of magnetic elements of one of the beam lines as a spectrometer for charge and mass selection.

The first set of main bends of the beam line are used to clean up the $\mathrm{Pb}^{82+}$ beam. There are contaminations of different species in the $\mathrm{Pb}^{82+}$ beam on the level of permille. The source of this contamination is mainly interaction in the steel of the beam splitters and in the material of the beam monitors. Using the first part of the beam line the contaminant species are identified and eliminated with a collimator located at a position of maximum dispersion. Thus a pure $\mathrm{Pb}^{82+}$ beam impinges on the target located in the middle of the transfer tunnel.

The second part of the beam line was used to analyse the different masses and charge states exiting the target. This was done by scanning a collimator slit across the beam phase space at a point which had a large momentum dispersion with a horizontal and vertical focus. The transmitted ions were counted in a Cerenkov counter at the end of the beam line. The momentum resolution of the system was $\sim 7 \times 10^{-4}$ and the different species could easily be separated and identified. 


\section{DISCLAIMER}

This report was prepared as an account of work sponsored by an agency of the United States Government. Neither the United States Government nor any agency thereof, nor any of their employees, make any warranty, express or implied, or assumes any legal liability or responsibility for the accuracy, completeness, or usefulness of any information, apparatus, product, or process disclosed, or represents that its use would not infringe privately owned rights. Reference herein to any specific commercial product, process, or service by trade name, trademark, manufacturer, or otherwise does not necessarily constitute or imply its endorsement, recommendation, or favoring by the United States Government or any agency thereof. The views and opinions of authors expressed herein do not necessarily state or reflect those of the United States Government or any agency thereof. 


\section{DISCLAIMER}

Portions of this document may be illegible in electronic image products. Images are produced from the best available original document. 


\section{RESULTS}

\section{a) Electron capture}

The cross section for electron loss is known to be several orders of magnitude bigger than the electron capture cross section. To separate the two cross sections it is necessary to measure the yield of $\mathrm{Pb}^{81+}$ ions as a function of different thicknesses for each target type. Beyond a certain target thickness, equilibrium between the number of $\mathrm{Pb}^{82+}$ and $\mathrm{Pb}^{81+}$ ions will be established. It is important to choose the thicknesses of the target such that the $\mathrm{Pb}^{81+}$ growth curve is sampled below the equilibrium. Figure 1 gives an example of the measured fraction of $\mathrm{Pb}^{81+}$ ions versus thickness for Au targets.

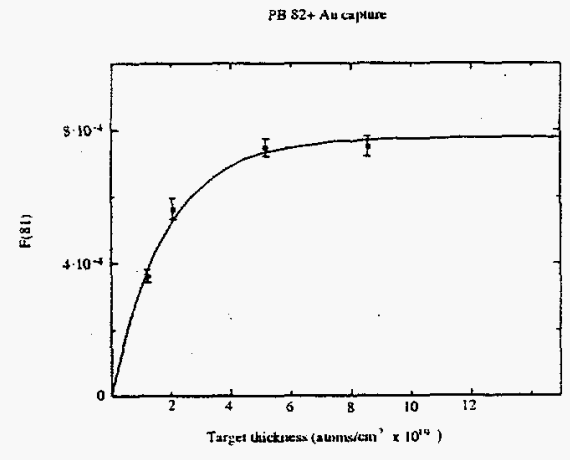

Figure 1: Fraction of $\mathrm{Pb}^{81+}$ ions for different thicknesses of Au targets.

The data of Figure 1 can be used to determine both the capture cross section $\left(\sigma_{c}\right)$ and the cross section for electron loss $\left(\sigma_{\mathrm{i}}\right)$ by fitting the data using a two-state model [6]:

Table 1:Capture $\left(\sigma_{c}\right)$ and electron loss $\left(\sigma_{1}\right)$ cross sections. $\sigma_{\mathrm{ECPP}}$ is derived from $\sigma_{c}$ (see text).

\begin{tabular}{|c|c|c|c|c|}
\hline Target & $\mathrm{Z}$ & $\sigma_{1}$ (kbarn) & $\sigma_{\text {(barn) }}$ & $\sigma_{\text {...me }}$ (barn) \\
\hline $\mathrm{Be}$ & 4 & 0.15 & 0.23 & 0.18 \\
\hline $\mathrm{C}$ & 6 & 0.31 & 0.44 & 0.36 \\
\hline $\mathrm{Al}$ & 14 & 1.4 & 1.6 & 1.5 \\
\hline $\mathrm{Ar}$ & 18 & 2.0 & 2.9 & 2.6 \\
\hline $\mathrm{Cu}$ & 29 & 8.0 & 7.2 & 6.8 \\
\hline $\mathrm{Kr}$ & 36 & 7.4 & 10.1 & 9.6 \\
\hline $\mathrm{Sn}$ & 50 & 21 & 19.2 & 18 \\
\hline $\mathrm{Xe}$ & 54 & 16 & 20.7 & 20 \\
\hline $\mathrm{Au}$ & 79 & 53 & 44.3 & 43 \\
\hline
\end{tabular}

As mentioned in the introduction three different reaction mechanisms contribute to the total capture cross section. Each of the processes has a different scaling behaviour relative to the target atomic number $\left(Z_{\mathrm{T}}\right)$ and the relativistic Lorentz factor of the incoming ion $(\gamma)$. The radiative capture scales as $Z_{\mathrm{T}} / \gamma$ and the non-radiative capture has a $\mathrm{Z}_{\mathrm{T}}^{3} / \gamma$ scaling. On the other hand, the capture from pair production scales as $Z_{\mathrm{T}}{ }^{2} \ln \gamma$ [5] which makes this process dominate at high enough energies. In order to separate the ECPP contribution, which is the only one relevant for estimation of lifetimes in colliders, the calculated REC and NRC contributions [7] have been subtracted from the measured total capture cross section. The ECPP cross sections obtained in this way are indicated in Table 1 and plotted in figure 2. A dependence close to $Z_{T}{ }^{2}$ is clearly seen.

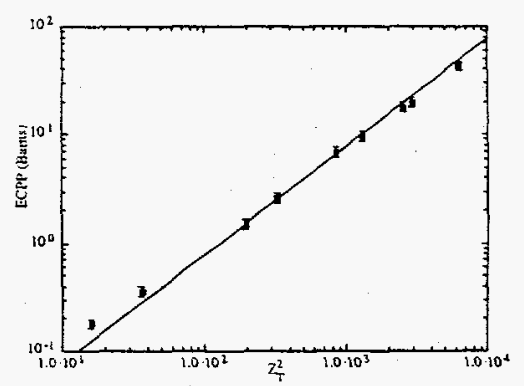

Figure 2: The measured $\sigma_{E C P P}$ cross sections as a function of $Z_{T}^{2}$.

\section{b) Electromagnetic dissociation}

In this case the full beam line optics and the slits were tuned for $\mathrm{Pb}^{82+}$ ions and the total cross section was determined from the measured attenuation in the target. The attenuation was obtained by integrating the transmitted beam intensities for a number of targets with different thicknesses

The total experimental cross sections obtained for the five targets are shown in Table 2.

The hadronic cross section and the electromagnetic cross section have very different energy and $Z$ behaviours. Thus, it is necessary to separate the two contributions in order to be able to make an extrapolation to higher energies and other species. This has been done by a theoretical estimate of the hadronic contribution [8]. The calculated values of $\sigma_{h}$ for the different targets are listed in Table 2. The table also lists a small contribution to the electromagnetic dissociation cross section from bound electrons in the target [8]. This contribution has to be subtracted as it will not be present in a collider. The last column of Table 2 lists the electromagnetic dissociation cross section from ion-ion interaction as obtained from our measurement, subtracting the hadronic and the small electron contribution.

Table 2: Measured total loss cross sections ( $\left.\sigma_{\text {meas }}\right)$.

\begin{tabular}{|c|c|c|c|c|c|}
\hline Target & $\mathbf{Z}$ & $\begin{array}{c}\sigma_{\text {mess }} \\
\text { (barn) }\end{array}$ & $\begin{array}{c}\sigma_{\mathrm{n}} \\
\text { (barn) }\end{array}$ & $\begin{array}{c}\sigma_{\text {e }} \\
\text { (barn) }\end{array}$ & $\sigma_{\text {das }}$ (barn) \\
\hline $\mathrm{C}$ & 6 & 4.5 & 3.4 & 0.051 & 1.05 \\
\hline $\mathrm{Al}$ & 14 & 7.4 & 4.3 & 0.13 & 2.97 \\
\hline $\mathrm{Cu}$ & 29 & 15.2 & 5.5 & 0.28 & 9.43 \\
\hline $\mathrm{Sn}$ & 50 & 31.0 & 6.6 & 0.48 & 23.9 \\
\hline $\mathrm{Pb}$ & 82 & 64.0 & 7.9 & 0.78 & 55.3 \\
\hline
\end{tabular}

\section{CONSEQUENCES FOR LHC AND RHIC}

The luminosity lifetime of high energy heavy ion collider is determined by the blow up of the beams due to the intra-beam scattering and the losses in the interaction(s) points due to beam-beam interactions. The intra-beam scattering is machine 
dependent while the losses due to interactions exclusively depend on the underlying physics processes. Here we will only consider the ultimate machine independent limit set by the physics processes.

To estimate the effect we have to extrapolate the relevant cross sections from SPS energies to RHIC and LHC energies. The Lorentz factor must be taken in a system where one of the nuclei is at rest and we get

$$
\gamma=2 \gamma_{\text {coll }}{ }^{2}-1
$$

The effective Lorentz factor $\gamma$ at RHIC and LHC with $100 \mathrm{GeV} / \mathrm{u}$ and $2.76 \mathrm{GeV} / \mathrm{u}$, respectively, becomes $\gamma_{\text {RHIC }}=2.310^{4}$ and $\gamma_{\text {LHC }}=1.710^{7}$. This should be compared to $\gamma=168$ at the SPS.

The electron capture from pair production cross section has been shown to scale as $A \ln \gamma+B$ [5] where $A$ and $B$ are independent of $\gamma$ at $\gamma=\sim 100$. Including both perturbative and nonperturbative contributions, $B$ is estimated to be -24 barn [9]. Applying this scaling to our measured cross section of $\mathrm{Pb}{ }^{82+}$ on Au from Table 1 and making a small correction for the Z-dependence going to a symmetric $\mathrm{Au}$ or $\mathrm{Pb}$ system we obtain $\sigma_{\text {capture }}$ RHIC $=94$ barn and $\sigma_{\text {capture }}{ }_{\text {LHC }}=204$ barn, respectively. For the target we applied a $Z_{\mathrm{T}}{ }^{2}$ scaling and a $\mathrm{Z}_{\mathrm{p}}^{5}$ behaviour was assumed for the projectile.

The scaling of the electromagnetic dissociation cross section is somewhat less straightforward. The strong electromagnetic field is simulated by an equivalent photon spectrum and the cross section is essentially determined by this spectrum folded with the photo-nucleon dissociation cross section. The calculation has to be done integrating over all photon energies. The energy dependence is slightly different depending on what part of the photon spectrum is considered [10]. The dominant contribution comes from the giant dipole resonance in the energy region up to $40 \mathrm{Mev}$ and this part has a simple $\ln \gamma$ scaling [10]. At SPS energies this part constitutes $85 \%$ of the total electromagnetic dissociation cross section [8]. Thus we have taken $85 \%$ of our measured cross section from Table 2 and scaled it to higher energies. For the remaining $15 \%$ we rely upon the calculation in Ref. [11] and adding this contribution we get $\sigma_{\text {dis }}{ }^{\text {RHIC }}=123$ barn and $\sigma_{\text {dis }}{ }^{\text {LHC }}=225$ barn.

The energy dependence of the hadronic cross section is taken from a dispersion relation calculation based on measurements of $\sigma_{\text {tot }}$ and of the parameter $p$ (ratio of real to imaginary part of the forward amplitude) for proton-proton interactions [12]. This calculation predicts an increase of the cross section by a factor 1.2 for RHIC and 2.4 for LHC relative to SPS energies. Assuming the same energy dependence for $\mathrm{Au}$ or $\mathrm{Pb}$ interactions we get $\sigma_{\text {thad }}^{\text {RHIC }}=9.5$ barn and $\sigma_{\text {laas }}^{\text {LHC }}=19$ barn.

Adding the three cross sections together, we get $\sigma_{\omega * i}{ }^{\text {RHIC }}=227$ barn and $\sigma_{\text {tot }}^{\text {LHC }}=448$ barn. It should be pointed out that these cross sections are somewhat larger than what was originally used for RHIC and LHC estimates.

The beam loss due to beam-beam interaction is proportional to $\sigma_{t 00}$, the instantaneous luminosity and the number of interaction points $\mathrm{n}$. With $\mathrm{N}$ being the number of ions in the beam we have:

$$
\mathrm{dN} / \mathrm{dt}=-\mathrm{L}(\mathrm{t}) \sigma_{\mathrm{tot}} \mathrm{n}
$$

Since $\mathrm{L}$ is proportional to $\mathrm{N}(\mathrm{t})^{2}$ we get as solution to (3)

$$
N(t)=N_{0} /\left(1+t / t_{b}\right) \text {, }
$$

where $t_{b}$ is given by

$$
t_{b}=N_{0} /\left(L_{0} \sigma_{t o t} n\right) \text {. }
$$

Defining the luminosity lifetime as if the decay were exponential we have

$$
t_{1 / 2}=\ln 2 / 2 t_{b} .
$$

Using the above formulae and taking the values of the initial luminosity and number of ions in the beam from the RHIC and LHC design reports $[1,2]$ we can calculate the luminosity half-life due to beam-beam interactions. We get a luminosity half-life of $7.3 \mathrm{hrs}$ at RHIC and $5.8 \mathrm{hrs}$ at LHC. These values are not far from those stated in the design reports which were $14 \mathrm{hrs}$ and $6.7 \mathrm{hrs}$, respectively. They also are quite similar to those calculated by Baltz et al. in Ref. [4]. These previous estimates were based upon extrapolations from low energy data in combination with theoretical considerations. Our measurement at SPS energies, where the $\ln \gamma$ scaling is supposed to be valid, basically confirms that the assumptions made earlier were reasonable.

\section{ACKNOWLEDGEMENT}

Authors (H.F. Krause, C.R. Vane and S. Datz) acknowledge support by the U.S. Department of Energy, Office of Basic Energy Sciences, Division of Chemical Sciences under contract No: DE-ACO5-96OR22464 with Lockheed Martin Energy Research Corporation. H. Knudsen and U. Mikkelsen acknowledge support of the Danish Natural Science Research Council. We also would like to acknowledge preparation of the solid targets used in the electron capture measurements to the GSI target shop.

\section{REFERENCES}

[1] LHC Conceptual Design, CERN/AC/95-05 (1995).

[2] RHIC Design Manual, BNL-52195 (1989).

[3] D. Brandt et al. CERN SL/94-04(AP).

[4] A.J. Baltz et al. Phys.Rev. E54, 4233 (1996).

[5] A.J. Baltz et al. Phys.Rev. A44, 5569 (1991).

[6] H.F. Krause et al. Phys. Rev. Lett. 80, 1190 (1998).

[7] R. Anholt and U. Becker, Phys.Rev. A36, 4628 (1987).

[8] S. Datz, et al. Phys. Rev. Lett. 79, 3355 (1997).

[9] A.J. Baltz et al. Phys. Rev. A50, 4842 (1994).

[10] G. Baur, C.A. Bertulani, Nucl. Phys. A505 835 (1989).

[11] M. Vidovic et al. Phys. Rev. C48, 2011 (1993).

[12]C. Augier et al., Phys. Lett. B316 (1993) 448. 